\title{
Lung cancer screening and smoking abstinence: 2 year follow-up data from the Dutch-Belgian randomised controlled lung cancer screening trial
}

\author{
Carlijn Michelle van der Aalst, ${ }^{1,2}$ Karien Anna Margaretha van den Bergh, ${ }^{1}$ \\ Marc Christiaan Willemsen, ${ }^{3}$ Henricus Johannes de Koning, ${ }^{1}$ \\ Robertus Johannes van Klaveren ${ }^{2}$
}

'Department of Public Health, Erasmus MC, University Medical Centre Rotterdam, Rotterdam, The Netherlands 2Department of Pulmonology, Erasmus MC, University Medical Centre Rotterdam, Rotterdam, The Netherlands

${ }^{3}$ STIVORO-Dutch Expertise Centre on Tobacco Control, The Hague, The Netherlands

Correspondence to

C M van der Aalst, Department of Public Health/Pulmonology, Erasmus MC, University Medical Centre Rotterdam, 3000 CA Rotterdam, The Netherlands; c.vanderaalst@erasmusmc.nl

NELSON is a Dutch abbreviation for Dutch-Belgian Lung cancer Screening Trial

(NEderlands-Leuvens Longkanker

ScreeningsONderzoek)

Received 18 December 2009 Accepted 9 April 2010

\section{ABSTRACT}

Background Lung cancer screening may provide a new opportunity for attempts to quit among smokers or might delay smoking cessation, but studies to date failed to provide evidence for this. This study investigated the effect of lung cancer screening on smoking abstinence in male smokers participating in the Dutch-Belgian randomised controlled lung cancer screening trial (NELSON trial).

Methods In the NELSON trial, 50- to 75-year-old participants at high risk for developing lung cancer were randomised to either lung cancer screening or no screening. Smoking behaviour was evaluated in two random samples of male smokers in the screen $(n=641)$ and control arm $(n=643)$ before (T0) and 2 years after randomisation (T1). In addition, the data were also analysed by intention-to-treat (ITT) analysis, as recommended in smoking cessation intervention trials, although non-response in screening trials can also be due to reasons other than continued smoking.

Results Almost 17\% (16.6\%) of the trial participants quit smoking, which is higher than the $3-7 \%$ found in the general adult population. However, screening was associated with a lower prolonged abstinence rate (14.5\%) compared with no screening (19.1\%) (OR 1.40, $95 \% \mathrm{Cl} 1.01$ to $1.92 ; p<0.05)$. No stastistically significant difference was found after performing an ITT analysis.

Conclusions This study showed that all trial participants were inclined to stop smoking more than average, which suggests that screening is a teachable moment to improve smoking behaviour. In those who underwent screening the smoking abstinence rate was significantly lower than for the control group, although the difference was modest. After ITT analysis this difference was no longer observed.

Clinical trial number ISRCTN63545820.

\section{INTRODUCTION}

Smoking is highly correlated with the development of lung cancer, the leading cause of cancer death worldwide. ${ }^{12}$ As $\sim 80-90 \%$ of all cases are attributable to smoking, ${ }^{2}$ the most effective way to reduce the risk for developing lung cancer substantially is to refrain from smoking. ${ }^{3}$ Clinically diagnosed lung cancer is often in an advanced stage and occurs more often in former than in current smokers today, which highlights the need for further secondary preventive measures in addition to smoking cessation. ${ }^{4}$ For that reason, in different randomised trials the cost-effectiveness of lung cancer screening by low-dose CT is being evaluated. ${ }^{5}$ Subjects who participated in a lung cancer CT screening trial showed a high interest in smoking cessation-related interventions, which provides new opportunities to approach this population for smoking cessation programmes. ${ }^{7-10}$ People eligible for lung cancer screening usually are of advanced age with a long and intensive smoking history and often smoking-related comorbid diseases. ${ }^{11} 12$ It is well known that these smokers are relatively less motivated to quit smoking and less often seek smoking cessation support, ${ }^{13}{ }^{14}$ even though smoking cessation could lead to significant health benefits in this population. ${ }^{15}$

In several observational studies, participation in a lung cancer screening programme was found to be associated with smoking abstinence, ${ }^{3} 916$ with cessation rates ranging between $7 \%$ and $23 \%,{ }^{79} 1718$ which is encouraging compared with a quit rate of between of $3 \%$ and $7 \%$ in the general adult population. ${ }^{19}$ Ostroff et al reported that $87 \%$ of the participants who changed their smoking behaviour stated that participation in the screening programme had been a major influence on their motivation to quit smoking. The studies reported on this topic so far are difficult to compare, ${ }^{79} 1018$ and one concern that remains is that lung cancer screening may act as a licence to smoke, because of the potential reassuring effect of screening. ${ }^{7} 918$

Only data from one randomised controlled trial (RCT) for lung cancer screening are available comprising 4104 participants (45\% women); in this trial Ashraf et al reported similar smoking behavioural changes in both trial arms after lung cancer screening. ${ }^{20}$

Our study is the first RCT on lung cancer screening that explored the smoking behaviour in both trial arms and where the control arm participants have never been invited to the screening site. The purpose of the study was to investigate the effect of lung cancer screening (screen arm) on smoking abstinence compared with no screening (control arm) among participants in the Dutch-Belgian randomised controlled lung cancer screening trial (NELSON trial) after 2 years of follow-up, and to identify the baseline characteristics associated with smoking abstinence.

\section{METHODS \\ Study design}

The study design of the NELSON trial has been described elsewhere. ${ }^{621}$ In summary, the volunteers 
who gave their informed consent (15 822) were randomised (1:1) to either the screen arm or the control arm. Participants in the screen arm received lung cancer screening according to the study protocol and the test result was based on a nodule management protocol. ${ }^{622}$ The participants in the control arm received usual care (no screening), without any invitation to the screening site. At randomisation, all current smokers in both study arms received a standard smoking cessation brochure or a questionnaire by which people could ask for tailored smoking cessation information from STIVORO, the Dutch expert centre on tobacco control. The standard brochure contained brief information about the advantages of quitting, the barriers to quitting, tips about how to quit smoking and how to prevent smoking relapse, and the possibilities for smoking cessation support. The questionnaire consisted of questions about smoking history, previous attempts to quit, attitude towards smoking cessation and self-efficacy in smoking abstinence.

The NELSON trial was approved by the Dutch Minister of Health after positive advice from the Dutch Health Council and by the Ethical Boards of the participating centres.

\section{Study population}

The NELSON trial

Information regarding the recruitment rounds and selection procedure of the NELSON population has also been described before. $^{6} 2122$ In brief, people aged between 50 and 75 years with a smoking history of $>15$ cigarettes a day for $>25$ years or $>10$ cigarettes a day for $>30$ years, and who were current smokers, or former smokers who quit smoking $<10$ years ago, were invited to participate in the NELSON trial. ${ }^{21}$

\section{The effect of lung cancer screening}

The current study was conducted in a random subgroup of current male smokers randomised to the screen $(n=641)$ or control $(n=643)$ arm of the NELSON trial during the first recruitment period (figure 1). A current smoker was defined as a participant who had smoked 7 days prior to completing the baseline questionnaire before randomisation (T0). Screened male smokers who received a positive scan result $(n=53(2.1 \%))$ or who were off-study $(n=163(6.3 \%)$ in the screen arm and $n=7$ $(0.3 \%)$ in the control arm) were excluded from this sample (figure 1$)$. The selected population $(n=1284)$ received a second questionnaire to measure smoking behaviour in November 2006, which was 2.2 (SD 0.29) years after randomisation (T1).

\section{General questionnaire (TO)}

The general questionnaire included demographic variables (date of birth, gender, level of education) and smoking-related variables. ${ }^{21}$ The intention to quit smoking (8-point scale) was adapted from the Transtheoretical Model and recoded according to the stages of change. Respondents with no intention of quitting smoking within 1 year were classified as immotive, whereas precontemplators, contemplators and preparators reported an intention to quit smoking within $6-12$ months, $1-6$ months or 1 month, respectively. ${ }^{23} 24$ Other smoking-related items were the age of smoking initiation (8-point scale); the average number of cigarettes smoked a day (10-point scale); the number of years of smoking (9-point scale); and the time to the first cigarette after waking up. These last variables were recoded to a variable with 4-5 categories and into a continuous variable based on the mean value of each category. The last item is a measure of nicotine

Figure 1 Study flow diagram.

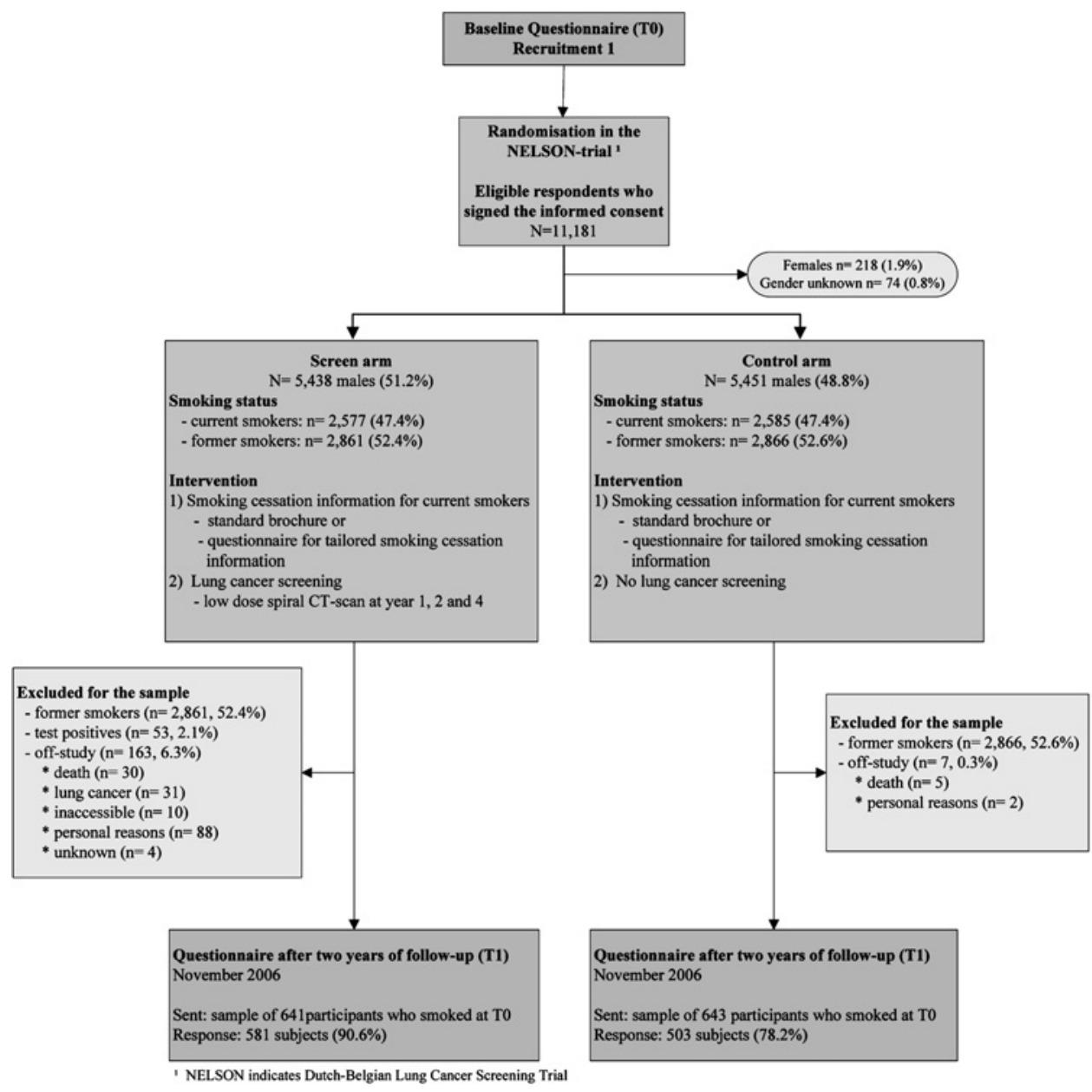


addiction and is adapted from the Fagerström Test for Nicotine Dependence (FTND). ${ }^{24} 25$

\section{Smoking cessation questionnaire (T1)}

In addition to the questions at $\mathrm{T} 0$, we asked participants about their marital status/home situation and their smoking behaviour. Current smoking behaviour was measured by questions regarding whether participants still smoked (yes/no); the number of cigarettes, shag (rolling tobacco), cigar/cigarillos and/ or pipe smoked a day; and whether they had smoked during the last $24 \mathrm{~h}$ (yes/no) and 7 days (yes/no) before completing the questionnaire. ${ }^{24}$ Respondents who answered that they still smoked or who smoked in the last 7 days were classified as current smokers. In all other cases, they were regarded as point prevalent smoking abstinent. In addition, these participants were asked the following questions. 'Are you engaged in an attempt to quit at this moment (yes/no)?' 'What was the date of the attempt to quit (day/month/year)?' 'Have you smoked since this quit date (not at all/ $1-5$ cigarettes $/>5$ cigarettes) and since 2 weeks after this quit date (not at all $/ 1-5$ cigarettes $/>5$ cigarettes)? 'How many attempts to quit lasting for at least $24 \mathrm{~h}$ have you made in the past? ${ }^{24}$ Former smokers who had smoked $<5$ cigarettes since 2 weeks after the quit date were classified as prolonged smoking abstinent, whereas former smokers who had smoked $<5$ cigarettes since the quit date were classified as continued smoking abstinent. All others were classified as current smokers all the time. ${ }^{24}$ Prolonged smoking abstinence was the primary outcome measure of this study. ${ }^{26}$ The selfreported smoking status was not biochemically verified.

To calculate the increase or reduction in smoking intensity, the numbers of cigarettes smoked at follow-up were recoded to the categories as measured at T0, which was the least exact measurement. Transitions through the categories were calculated; backward change was classified as reduced smoking, whereas forward change was classified as increased smoking. Otherwise, the smoking behaviour was defined as stable.

\section{Statistical analyses}

Power analysis indicated that a sample of 480 participants in the screen arm and 240 participants in the control arm would have $80 \%$ power to detect an expected difference in quit rates of $14 \%$ in the screen arm and $7 \%$ in the control arm. Similarities in distribution of the baseline characteristics in both trial arms were analysed using Pearson $\chi^{2}$ statistics for categorical variables and the Mann-Whitney U test for continuous variables with a nonnormal distribution. These tests were also used to explore differences in smoking behaviour in participants in the screen arm and control arm at T1. Both univariate and multivariate (backward) logistic regression analyses were performed to investigate whether baseline characteristics predict prolonged smoking abstinence at T1. In addition, the association between lung cancer screening and smoking abstinence was calculated by the 'intention-to-treat' (ITT) method (worst-case scenario) as recommended for smoking cessation intervention studies. ${ }^{26}$ According to this method, nonresponders are considered as current smokers.

The level of significance was set at 0.05 (two-tailed). The statistical package software $\mathrm{R}$ was used for the power analysis and all other statistics were performed using SPSS version 15.0.

\section{RESULTS}

\section{Baseline characteristics of the participants}

The response rates to the questionnaires were 90.6\% (581/641) and $78.2 \%(503 / 643)$ for the screen arm and control arm, respectively (figure 1). The mean age of the respondents $(\mathrm{n}=1084)$ was 58 (IOR 7) years. Moreover, 892 of the 1084 participants (82.3\%) lived together and 504 out of 1067 participants $(47.2 \%)$ had a lower education level. The majority of the respondents had a smoking history of 31-50 pack-years (561/ $1084 ; 51.8 \%$ ) and started smoking at the age of $15-20$ years $(704 / 1084 ; 64.9 \%)$. One hundred and seventy-nine smokers $(179 / 1020 ; 17.5 \%)$ reported starting smoking $<5 \mathrm{~min}$ after waking up, which reflects nicotine addiction. At T0, 40.8\% (432/ 1058) of the smokers did not intend to stop smoking, while $15.6 \%$ (165/1058), $28.9 \%(306 / 1058)$ and $14.7 \%(155 / 1058)$ of the smokers had the intention to stop within 1 year (precontemplation stage of quitting), 6 months (contemplation stage) or 1 month (preparation stage), respectively (table 1).

The baseline characteristics of the respondents to the subcohort questionnaire were comparable with the baseline characteristics of male smokers in the NELSON trial of the first recruitment round (table 1). Furthermore, responders from the screen arm and control arm (table 1), and responders and nonresponders (data not shown) had similar baseline characteristics (no statistically significant differences).

\section{Lung cancer screening and smoking behaviour}

The smoking behaviour of the responders after 2 years of participation in the screening trial (T1) is presented in table 2. No difference was found in the number of attempts to quit between the screen arm and control arm participants. At T1, respondents in the control arm reported a significantly higher point prevalence of smoking abstinence (OR 1.38; 95\% CI 1.01 to 1.90 ), as well as a lower prolonged (OR 1.40; $95 \%$ CI 1.01 to 1.92 ) and continued abstinence rate (OR 1.42; 95\% CI 1.03 to 1.96) compared with the screen arm.

According to the ITT analysis, assuming that the nonrespondents were current smokers, the point prevalence of smoking abstinence was $13.7 \%$ (88/641) and 15.5\% (99/640) in the screen arm and control arm, respectively, after 2 years of follow-up ( $p=0.38)$. The prolonged and continued abstinence rates were, respectively, $13.1 \%(84 / 641)$ and $12.6 \%(81 / 641)$ in the screen arm and $14.9 \%(96 / 643)$ and $14.6 \%(94 / 643)$ in the control arm ( $\mathrm{p}=0.35$ and $\mathrm{p}=0.30$ ).

Complete data for the abstinence period were available for $75 \%$ (63/84) of the screened participants and $72 \%$ (69/96) of the control arm participants (table 2). At T1, the median period of prolonged abstinence was 12.0 months in both trial arms. After 2 years of follow-up, the current smokers in the screen $(\mathrm{n}=497)$ and control $(\mathrm{n}=407)$ arm smoked 20 (IOR, 13-12, respectively) cigarettes a day $(\mathrm{p}=0.90)$. A similar proportion of current smokers in the screen $(264 / 497 ; 53.1 \%)$ and control $(219 / 407 ; 53.8 \%)$ arm reported having reduced their smoking intensity $(\mathrm{p}=0.23)$. In addtion, $17.7 \%$ (88/497) and $13.8 \%(56 / 407)$ of the smokers increased their smoking intensity and 29.2\% (145/497) and 32.4\% (132/407) remained stable in the screen and control arm, respectively.

\section{Predictors of prolonged smoking abstinence}

Univariate baseline characteristics associated with prolonged smoking abstinence at T1 were a higher educational level $(p=0.01)$, an intention to quit smoking within $1-6$ months and within 1 year $(p=0.01)$, allocation to the control arm $(p=0.04)$ and a time to the first cigarette of $>60 \mathrm{~min}$ and $30-60 \mathrm{~min}$ $(p=0.05)$ (table 3$)$. The age, the age at starting smoking, the number of cigarettes smoked a day or the smoking duration were not statistically significant predictors of prolonged smoking abstinence at follow-up (table 3).

Multivariate analysis (table 3) showed that only an intention to quit smoking within $1-6$ months $(p=0.03)$, a higher 
Table 1 Baseline characteristics of the participants

\begin{tabular}{|c|c|c|c|}
\hline & $\begin{array}{l}\text { Male smokers randomised in the } \\
\text { NELSON trial (first recruitment) } \\
\text { Total }(n=5161) \\
N(\%)^{*}\end{array}$ & \multicolumn{2}{|c|}{$\begin{array}{l}\text { Male smokers who responded to the smoking } \\
\text { cessation questionnaire }\end{array}$} \\
\hline \multicolumn{4}{|l|}{ Level of education $†$} \\
\hline Low educational level & $2442(48.0)$ & $280(49.3)$ & $224(44.9)$ \\
\hline Medium educational level & $1244(24.5)$ & $136(23.9)$ & $122(24.4)$ \\
\hline Married or living together & NA & $475(81.8)$ & 417 (82.9) \\
\hline \multicolumn{4}{|l|}{ Pack-years } \\
\hline$\leq 30$ & $1207(23.4)$ & $186(32.0)$ & $144(28.6)$ \\
\hline $31-40$ & $1810(35.1)$ & $168(28.9)$ & $161(32.0)$ \\
\hline $41-50$ & $1143(22.2)$ & $116(20.0)$ & $116(23.1)$ \\
\hline $51-60$ & $540(10.5)$ & $66(11.4)$ & $47(9.3)$ \\
\hline$>20$ years & $955(18.5)$ & $102(17.5)$ & $99(19.7)$ \\
\hline \multicolumn{4}{|l|}{ Intention to quit smoking $\ddagger(\mathrm{TO})$} \\
\hline Immotive & $2003(39.9)$ & $229(40.6)$ & $203(41.1)$ \\
\hline Precontemplator & $769(15.3)$ & $84(14.9)$ & $81(16.4)$ \\
\hline Contemplator & $1504(30.0)$ & $173(30.7)$ & $133(26.9)$ \\
\hline Preparator & $743(14.8)$ & 78 (13.8) & $77(15.6)$ \\
\hline \multicolumn{4}{|l|}{ Time to the first cigarette§ } \\
\hline$\leq 5 \min$ & $963(19.7)$ & $99(18.1)$ & $80(16.9)$ \\
\hline $5-30 \mathrm{~min}$ & $2000(40.8)$ & $206(37.8)$ & $205(43.2)$ \\
\hline $30 \mathrm{~min}-1 \mathrm{~h}$ & $1217(24.9)$ & $146(26.7)$ & $120(25.3)$ \\
\hline$>1 \mathrm{~h}$ & $717(14.6)$ & $95(17.4)$ & $69(14.6)$ \\
\hline
\end{tabular}

*Available data are presented as $\mathrm{N}(\%)$ unless indicated otherwise.

tLow educational level indicates primary, lower secondary general or lower vocational education; medium educational level, intermediate vocational education or higher secondary education high educational level, higher vocational education or university.

łImmotive indicates no intention to quit smoking within 1 year; precontemplator, intention to quit smoking within 1 year, but not within 6 months; contemplator, intention to quit smoking within 6 months, but not within 1 month; preparator, intention to quit smoking within the next month.

$\S$ First question of the Fagerström test for Nicotine Dependence (FTND).

NA, not applicable.

educational level $(\mathrm{p}=0.04)$ and allocation to the control arm $(\mathrm{p}=0.04)$ were significantly associated with prolonged smoking abstinence at follow-up.

\section{DISCUSSION}

The high smoking abstinence rate observed among those screened $(14.5 \%)$ has also been reported in previous observational studies. ${ }^{7-10} 1820$ This abstinence rate is positive in comparison with the $3-7 \%$ quit rates observed in adults of the general population after a minimal intervention for smoking cessation. ${ }^{19}$ This is very encouraging, since screening trial participants are usually elderly people with a long and intense smoking history for whom it is difficult to make an attempt to quit. ${ }^{11} 14$ Despite this high abstinence rate among those screened, control arm participants reported modest but significantly higher smoking abstinence rates.

In contrast to our findings, Ashraf et al found no effect of the allocation to the screen or control arm on smoking habits, ${ }^{20}$ which could probably be explained by the fact that both screen and control arm participants were invited to the screening site for spirometry and minimal smoking cessation counselling offered by a specialised nurse each year. This might have mitigated the effect of CT screening on smoking behaviour, and could explain the lack of any difference in smoking behaviour

Table 2 Smoking behaviour of male smokers in the screen and control arm after 2 years of follow-up

\begin{tabular}{|c|c|c|c|c|c|}
\hline & Screen arm \%* & $\mathbf{N}$ & Control arm \%* & $\mathbf{N}$ & p Value \\
\hline Median number of quit attempts (IQR) $\dagger$ & $1(2)$ & 581 & 1(2) & 503 & 0.47 \\
\hline Point prevalence of smoking abstinence & 15.1 & $88 / 581$ & 19.8 & $99 / 500$ & 0.04 \\
\hline Prolonged smoking abstinence§ & 14.5 & $84 / 581$ & 19.1 & $96 / 503$ & 0.04 \\
\hline $\begin{array}{l}\text { Median duration of smoking cessation** } \\
\text { (IQR) (months) }\end{array}$ & $12.0(17.0)$ & 63 & $12.0(15.5)$ & 69 & 0.82 \\
\hline
\end{tabular}

\footnotetext{
*Data are presented as a percentage unless indicated otherwise.

†Results are based on available data of respondents who were current smokers at follow-up (T1).

$\ddagger$ Point prevalence of smoking abstinence indicates that respondents did not smoke in the last 7 days.

§Prolonged smoking abstinence indicates that respondents have smoked $<5$ cigarettes since 2 weeks after the quit date.

$\lceil$ Continued smoking abstinence indicates that respondents have smoked $<5$ cigarettes since the quit date.

${ }^{* *}$ Results are based on available data of respondents who were former smokers at follow-up (T1).
} 
Table 3 OR of baseline characteristics for prolonged smoking abstinence in male smokers 2 years after randomisation

\begin{tabular}{|c|c|c|}
\hline & \multicolumn{2}{|c|}{ Prolonged smoking abstinence } \\
\hline & $\begin{array}{l}\text { OR (95\% Cl), univariate } \\
\text { analysis }\end{array}$ & $\begin{array}{l}\text { OR }(95 \% \mathrm{Cl}), \\
\text { multivariate analysis }\end{array}$ \\
\hline \multicolumn{3}{|l|}{ Study arm } \\
\hline Screen arm & 1.00 & 1.00 \\
\hline Control arm & $1.40(1.01 \text { to } 1.92)^{*}$ & $1.45(1.02 \text { to } 2.04)^{*}$ \\
\hline Age (T0) & $0.99(0.96$ to 1.03$)$ & \\
\hline \multicolumn{3}{|l|}{ Level of education $\dagger$} \\
\hline Lower education & 1.00 & 1.00 \\
\hline Medium education & $1.27(0.83$ to 1.93$)$ & $1.29(0.84$ to 2.00$)$ \\
\hline Higher education & $1.84(1.26 \text { to } 2.67)^{*}$ & $1.66(1.12 \text { to } 2.48)^{*}$ \\
\hline \multicolumn{3}{|l|}{ Age at starting smoking } \\
\hline$<15$ years & 1.00 & \\
\hline $16-19$ years & 1.20 (0.77 to 1.87$)$ & \\
\hline$>20$ years & 0.73 (0.41 to 1.31$)$ & \\
\hline No. of cigarettes smoked a day & $0.98(0.96$ to 1.00$)$ & \\
\hline Smoking duration (years) & $1.00(0.97$ to 1.03$)$ & \\
\hline \multicolumn{3}{|l|}{ Time to the first cigarette $\neq$} \\
\hline$<5 \min$ & 1.00 & \\
\hline $5-30 \mathrm{~min}$ & $1.68(0.97$ to 0.93$)$ & \\
\hline $30-60 \mathrm{~min}$ & $1.97(1.10 \text { to } 3.51)^{*}$ & \\
\hline$>60 \min$ & $2.34(1.26 \text { to } 4.33)^{*}$ & \\
\hline \multicolumn{3}{|l|}{ Intention to stop smoking (TO)§ } \\
\hline Immotive & 1.00 & 1.00 \\
\hline Precontemplator & $1.65(1.02 \text { to } 2.69)^{*}$ & $1.60(0.98$ to 2.61$)$ \\
\hline Contemplator & $1.93(1.30-2.87)^{*}$ & $1.81(1.20 \text { to } 2.73)^{*}$ \\
\hline Preparator & $1.58(0.96$ to 2.60$)$ & $1.11(0.61$ to 2.00$)$ \\
\hline
\end{tabular}

*Statistically significant $O R(p<0.05)$.

†Low educational level indicates primary, lower secondary general or lower vocational education; medium educational level, intermediate vocational education or higher secondary education; high educational level, higher vocational education or university

\#First question of the Fagerström Test for Nicotine Dependence (FTND).

SImmotive indicates no intention to quit smoking within 1 year; precontemplator, intention to quit smoking within 1 year, but not within 6 months; contemplator, intention to quit smoking within 6 months, but not within 1 month; preparator, intention to quit smoking within the next month.

changes between both trial arms. ${ }^{10}$ The strengths of our study are that all smokers in our study received limited written smoking cessation information only once at randomisation, and that control arm participants were never invited to the screening site.

Data analysis according to the ITT method is generally recommended for the evaluation of smoking cessation intervention studies. ${ }^{26}$ Ashraf et al also used this method to analyse their data. ${ }^{20}$ When we applied this method, we also found no statistically significant difference in smoking abstinence between the screen and control arm. However, we believe that it is appropriate to exclude non-responders, because non-response of NELSON participants can reasonably also be explained by reasons other than continued smoking, such as loss of interest in screening for lung cancer. Furthermore, people are more likely to under-report their smoking intensity rather than their smoking status. ${ }^{27}$ Therefore, we believe that despite the lower response rate observed in the screen arm, allocation to the screen arm may lead to lower quit rates as compared with the control arm. Our concern is, therefore, that screening may create some relief among smokers based on false confidence. ${ }^{7}$ This unfavourable effect of screening on smoking cessation has not been reported in the context of lung cancer screening before. Only Larsen et al reported less improvement in smoking habits among screened individuals in a colorectal cancer screening trial. ${ }^{28}$ Furthermore, we found that half of the current smokers reduced their smoking intensity and that the other half did not change or even increased their smoking intensity after lung cancer screening. We should realise, however, that we were able to detect only large changes in smoking intensity, because of the wide categories used and because in reality even more people might have increased their smoking intensity.

These results emphasise the need to improve smoking habits in lung cancer screening programmes. So far, there is no evidence-based approach to how to integrate the promotion of the abstinence from smoking in lung cancer screening programmes. More research is warranted to identify the most cost-effective intervention and the best method to frame the intervention in lung cancer screening programmes. Important issues to explore are the best type of intervention, the optimum teachable moment(s) and whether the test result could be used as biofeedback to enhance quitting smoking.

A limitation of our study is that the data originate from selfcompleted questionnaires without biochemical verification of the smoking status, with the risk of social desirability response bias. However, self-reports on smoking behaviour appeared to be valid in a lung cancer screening setting. ${ }^{29}$ The recruitment was based on population registries, but randomised people volunteered to participate in the lung cancer screening trial. These volunteers were possibly more motivated to quit smoking compared with the general adult population. ${ }^{13}$ We excluded male smokers randomised to the screen arm with a positive test result from the sample that was selected for the substudy, because of the low prevalence $(2.1 \%)$ of this test result in the study population after the introduction of the indeterminate test result by the NELSON trial. This probably may have caused a small underestimation of the smoking cessation rate in participants in the screen arm, because a positive test result might motivate subjects to quit smoking. ${ }^{10} 1820$ When we adjust for the exclusion of the people with a positive test result ${ }^{20}$ the prolonged smoking abstinence rate in the screen arm was comparable and had no impact on the results. Another limitation is that this substudy was restricted to men, but, based on past research, we presume that the effect of lung cancer screening is similar for males and females at high risk for developing lung cancer. ${ }^{10} 1820$

Although we demonstrated an association between lung cancer screening participation and smoking abstinence, more research is warranted that explores whether this relationship is causal, because few available studies are available so far.

This study showed that all trial participants were inclined to stop smoking more than average, which suggests a teachable moment to improve smoking behaviour. In those who underwent screening the smoking abstinence rate was significantly lower than for the control group, although the difference was modest. After ITT analysis, this difference was no longer observed.

Acknowledgements We would like to thank R Faber and F Santegoets (Erasmus MC, Rotterdam, The Netherlands) for the data management, and A C de Jongh (Artex B.V. Capelle a/d IJssel, The Netherlands) for sending the questionnaires.

Funding The Netherlands Organization for Health Research and Development (ZonMW), the Dutch Cancer Association (KWF) and the Health Insurance Innovation Foundation (Innovatiefonds Zorgverzekeraars), The Netherlands. The funding sources had no involvement in the collection, analysis and interpretation of data; in the writing of the report; and in the decision to submit the paper for publication.

\section{Competing interests None}

Ethics approval This study was conducted with the approval of the Dutch Minister of Health after positive advice from the Dutch Health Council and by the Ethical Boards of the participating centres.

Provenance and peer review Not commissioned; externally peer reviewed. 


\section{REFERENCES}

1. Jemal A, Siegel R, Ward E, et al. Cancer statistics, 2008. CA Cancer J Clin 2008;58:71-96

2. WHO. WHO report on the global tobacco epidemic, 2008; the MPOWER package Geneva: World Health Organization, 2008.

3. Burns DM. Tobacco-related diseases. Semin Oncol Nurs 2003;19:244-9.

4. Jemal A, Siegel R, Ward E, et al. Cancer statistics, 2007. CA Cancer J Clin 2007:57:43-66

5. Field JK, Duffy SW. Lung cancer screening: the way forward. $\mathrm{Br} J$ Cancer 2008;99:557-62.

6. Van Klaveren RJ, Oudkerk M, Prokop M, et al. Management of lung nodules detected by volume CT scanning. N Engl J Med 2009;361:2221-9.

7. Ostroff JS, Buckshee N, Mancuso CA, et al. Smoking cessation following CT screening for early detection of lung cancer. Prev Med 2001;33:613-21.

8. Schnoll RA, Bradley P, Miller SM, et al. Psychological issues related to the use of spiral CT for lung cancer early detection. Lung Cancer 2003;39:315-25.

9. Taylor KL, Cox LS, Zincke N, et al. Lung cancer screening as a teachable moment for smoking cessation. Lung Cancer 2007; 56:125-34.

10. Townsend CO, Clark MM, Jett JR, et al. Relation between smoking cessation and receiving results from three annual spiral chest computed tomography scans for lung carcinoma screening. Cancer 2005;103:2154-62.

11. Alberg AJ, Ford JG, Samet JM. American College of Chest Physicians. Epidemiology of lung cancer: ACCP evidence-based clinical practice guidelines. (2nd edition). Chest 2007;132(3 Suppl):29S-55S.

12. U.S. Department of Health and Human Services. The health consequences of smoking: a report of the Surgeon General. Atlanta, GA: U.S. Department of Health and Human Services, Centers for Disease Control and Prevention, National Center for Chronic Disease Prevention and Health Promotion, Office on Smoking and Health, 2004. http://www.cdc.gov/tobacco/data_statistics/sgr/2004/complete_report/index. htm (accessed 20 2008).

13. Stivoro. [Smoking, the plain facts: adults 2006] Roken, de harde feiten: Volwassen 2006. Den Haag: Stivoro-voor een rookvrije toekomst [STIVORO-for a smokefree future], 2006. http://www.stivoro.nl/Upload/artikel/Cijfers/factsheet\% 20volwassenen2006\%203-09.pdf (accessed 22 Feb 2008).

14. Emmons KM. A research agenda for tobacco control. Cancer Causes Control 2000;11:193-4

15. Taylor DH Jr, Hasselblad V, Henley SJ, et al. Benefits of smoking cessation for longevity. Am J Public Health 2002;92:990-6.
16. Haussmann HJ. Smoking and lung cancer: future research directions. Int J Toxicol 2007:26:353-64

17. Clark MM, Cox LS, Jett JR, et al. Effectiveness of smoking cessation selfhelp materials in a lung cancer screening population. Lung cancer 2004:44:13-21.

18. Cox LS, Clark MM, Jett JR, et al. Change in smoking status after spiral chest computed tomography scan screening. Cancer 2003;98:2495-501.

19. Willemsen MC, Wagena EJ, van Schayck CP. [The efficacy of smoking cessation methods available in the Netherlands: a systematic review based on Cochrane data] Ned Tijdschr Geneeskd 2003;147:922-7.

20. Ashraf $\mathbf{H}$, Tonnesen $\mathrm{P}$, Pedersen $\mathrm{JH}$, et al. Smoking habits were unaffected by CT screening at 1-year follow-up in the Danish Lung Cancer Screening Trial (DLCST). Thorax 2009:64:388-92.

21. Van lersel CA, De Koning HJ, Draisma G, et al. Risk-based selection from the general population in a screening trial: selection criteria, recruitment and power for the Dutch-Belgian randomised lung cancer multi-slice CT screening trial (NELSON) Int J Cancer 2007:120:868-74.

22. Xu DM, Gietema $\mathrm{H}$, de Koning $\mathrm{H}$, et al. Nodule management protocol of the NELSON randomised lung cancer screening trial. Lung Cancer 2006;54:177-84.

23. DiClemente CC. Prochaska JO, Fairhurst SK, et al. The process of smoking cessation: an analysis of precontemplation, contemplation, and preparation stages of change. J Consult Clin Psychol 1991;59:295-304.

24. Mudde AN, Willemsen MC, Kremers S, et al. Measuring instruments for research regarding smoking and smoking cessation [Meetinstrumenten voor onderzoek naar roken en stoppen met roken.]. The Hague: STIVORO, 2006.

25. Heatherton TF, Kozlowski LT, Frecker RC, et al. The Fagerstrom Test for Nicotine Dependence: a revision of the Fagerstrom Tolerance Questionnaire. Br J Addict 1991:86:1119-27.

26. West $\mathbf{R}$, Hajek $\mathrm{P}$, Stead L, et al. Outcome criteria in smoking cessation trials proposal for a common standard. Addiction. 2005;100:299-303.

27. Patrick DL, Cheadle A, Thompson DC, et al. The validity of self-reported smoking: a review and meta-analysis. Am J Public Health 1994;84:1086-93.

28. Larsen IK, Grotmol T, Almendingen K, et al. Impact of colorectal cancer screening on future lifestyle choices: a three-year randomized controlled trial. Clin Gastroenterol Hepatol 2007:5:477-83

29. Studts JL, Ghate SR, Gill JL, et al. Validity of self-reported smoking status among participants in a lung cancer screening trial. Cancer Epidemiol Biomarkers Prev 2006;15:1825-8. 